PROCEEDINGS OF THE

AMERICAN MATHEMATICAL SOCIETY

Volume 131, Number 12, Pages 3945-3954

S 0002-9939(03)06964-8

Article electronically published on April 30, 2003

\title{
TOTALLY KNOTTED SEIFERT SURFACES WITH ACCIDENTAL PERIPHERALS
}

\author{
MAKOTO OZAWA AND YUKIHIRO TSUTSUMI
}

(Communicated by Ronald A. Fintushel)

\begin{abstract}
We show that if there exists an essential accidental surface in the knot exterior, then a closed accidental surface also exists. As its corollary, we know boundary slopes of accidental essential surfaces are integral or meridional. It is shown that an accidental incompressible Seifert surface in knot exteriors in $S^{3}$ is totally knotted. Examples of satellite knots with arbitrarily high genus Seifert surfaces with accidental peripherals are given, and a Haken 3-manifold which contains a hyperbolic knot with an accidental incompressible Seifert surface of genus one is also given.
\end{abstract}

\section{INTRODUCTION}

For a properly embedded surface $S$ in a 3-manifold $M$, a non-trivial loop $l$ in $S$ is called an accidental peripheral if $l$ is freely homotopic into $\partial M$ in $M$ but not in $S$, and $S$ having an accidental peripheral is said to be accidental. An annulus $A$ such that $A \cap S=\partial A \cap S=l$ and $\partial A=l \cup l^{\prime}$ where $l^{\prime}$ is an essential loop in $\partial M$, is called an accidental annulus.

Let $K$ be a knot in the 3 -sphere $S^{3}$. We denote the knot exterior $S^{3}-\operatorname{int} N(K)$ by $E(K)$. If $S$ is a Seifert surface bounded by $K$, we denote $S \cap E(K)$ by the same symbol $S$ and if it is accidental, we say the Seifert surface $S$ is accidental.

Fenley ([2]) proved that there exists no accidental Seifert surface of minimal genus by using the existence of a good Reebless foliation with that surface as a compact leaf ([3]). As its corollary, Fenley showed by using Thurston's result ([9]) that for any non-fibered hyperbolic knot, any lift of a minimal Seifert surface to the universal cover is a quasi-disk and its limit set is a quasi-circle in the sphere at infinity. Thus, if a non-fibered hyperbolic knot $K$ bounds an incompressible Seifert surface $S$ which does not have embedded accidental peripherals, then $S$ corresponds to a quasi-Fuchsian subgroup ([9]).

Question 1.1. Does there exist a knot which bounds an accidental incompressible Seifert surface?

Received by the editors July 25, 2000 and, in revised form, July 25, 2002.

2000 Mathematics Subject Classification. Primary 57M25; Secondary 57N10.

Key words and phrases. Accidental peripheral, quasi-Fuchsian, Seifert surface, totally knotted.

The first author was supported in part by Fellowship of the Japan Society for the Promotion of Science for Japanese Junior Scientists. 
Remark 1.2. The condition "incompressible" is necessary. In fact, any knot bounds an accidental compressible Seifert surface. Indeed, one can construct an accidental Seifert surface by tubing any Seifert surface and a narrow torus parallel to the knot.

In this paper, we prove that a large class of knots denies Question 1.1 and that some satellite knots bound accidental incompressible Seifert surfaces (Theorem 1.5).

Here, we remark that an existence of an accidental peripheral implies an existence of an embedded accidental annulus. In fact, if $S$ has an accidental peripheral, then Annulus theorem ([6]) gives an accidental peripheral with an embedded accidental annulus (Lemma 2.1).

For a non-peripheral closed incompressible surface $F$ embedded in $E(K)$ with an accidental annulus $A$, the slope of $A \cap \partial E(K)$ is called an accidental slope. It is known ([1]) that an accidental slope of a closed incompressible surface is an integer or $1 / 0$, and it was shown that $F$ has a unique accidental slope. Furthermore if the accidental slope is integral, its accidental annulus is unique up to isotopy ([5]).

Theorem 1.3 (Existence of a closed accidental surface). Let $K$ be a knot in $S^{3}$. If $E(K)$ contains an accidental essential surface with boundary slope $\gamma$, then $E(K)$ contains a closed accidental incompressible surface with accidental slope $\gamma$.

By Theorem 1.3 and 1 Lemma 2.5.3], the following theorem holds.

Theorem 1.4 (Integral or meridional). The boundary slope of an accidental essential surface is an integer or $1 / 0$.

In [5], it is conjectured that the integral accidental slope is unique for all accidental incompressible closed surfaces in $E(K)$. By Theorem 1.3 if this conjecture is true, we can conclude that the integral accidental boundary slope is unique.

A Seifert surface $S$ is said to be totally knotted if the exterior $S^{3}-\operatorname{int} N\left(S ; S^{3}\right)$ is $\partial$-irreducible. We say that $K$ is totally knotted if $K$ bounds a totally knotted Seifert surface. Notice that there exists a knot which does not bound a totally knotted Seifert surface.

For example, if $K$ is a fibered knot, then for an incompressible Seifert $F$, the exterior $S^{3}-\operatorname{int} N\left(F ; S^{3}\right)$ is a handlebody which is a product $F \times I$, so it is not totally knotted.

As will be shown later, an accidental incompressible Seifert surface is totally knotted, hence the remaining case for Question 1.1 is of non-minimal, totally knotted Seifert surfaces. We can also show that totally knotted Seifert surfaces with some conditions are not accidental.

These arguments will be given in section 3 . However, there exists a satellite knot with totally knotted, non-minimal genus accidental Seifert surfaces.

Theorem 1.5 (Accidental incompressible Seifert surfaces). There exist infinitely many genus one satellite knots, each of which bounds an accidental incompressible Seifert surface of arbitrarily high genus.

This theorem gives a positive answer for Question 1.1. If the knot exterior $E(K)=S^{3}-\operatorname{int} N(K)$ contains no essential torus, Thurston's geometrization theorem assures that $E(K)$ is a Seifert manifold or $S^{3}-K$ admits a complete hyperbolic structure of finite volume. It is known that for a Seifert manifold with non-empty boundary, closed incompressible surface is isotopic to a torus which is a union of fibers ([6]). Hence, if $E(K)$ is a Seifert manifold, $K$ does not bound a totally knotted Seifert surface. 
In section 4, we will construct a closed hyperbolic Haken 3-manifold which contains a hyperbolic knot with an accidental incompressible Seifert surface of genus one. Indeed our examples of Theorem 1.5 are satellite, namely, contain essential tori in exteriors; we could construct hyperbolic examples in arbitrary 3-manifolds as confirmed by J. Weeks' computer program 'SnapPea'.

\section{Boundary slopes of ESSENTIAL ACCIDENTAL SURFACES}

In this section, we consider the existence of embedded accidental annulus and prove Theorem 1.3. Hereafter, all 3-manifolds are assumed to be orientable. For a surface $S$ properly embedded in a 3 -manifold $M$, we denote the regular neighborhood of $S$ in $M$ by $N(S ; M)$, or simply $N(S)$. We denote the frontier of $N(S ; M)$ by $\partial N(S ; M)$, and let $\operatorname{int} N(S ; M)$ denote the topological interior of $N(S ; M)$ in $M$.

Lemma 2.1 (Embedded accidental annulus). Let $S$ be a two-sided surface properly embedded in a compact, irreducible, $\partial$-irreducible 3-manifold $M$ with $\partial M$ a union of some tori. If $S$ is incompressible and $\partial$-incompressible in $M$ and has an accidental peripheral, then there exists an embedded accidental annulus for $S$.

Proof. Since $S$ is accidental, there exists a map $f: S^{1} \times[0,1] \rightarrow M$ generic to $S$ such that $f\left(S^{1} \times\{0\}\right)$ is an accidental peripheral $l$ and $f\left(S^{1} \times\{1\}\right) \subset \partial M$. By the hypothesis that $S$ is two-sided incompressible and $\partial$-incompressible, we have that $\partial N(S)$ is incompressible and $\partial$-incompressible in $M$-int $N(S)$. Using the product structure of $N(S)$ and the incompressibility and the $\partial$-incompressibility of $\partial N(S)$, we can modify $f$ so that $f^{-1}(S)$ contains only essential embedded loops in $S^{1} \times[0,1]$ and we may assume that $\left|f^{-1}(S)\right|$ is minimal among all accidental peripherals and such maps. Let $A$ be the closure of a component of $S^{1} \times[0,1]-f^{-1}(S)$ such that $S^{1} \times\{1\} \subset \partial A$. If $f\left(\partial A-S^{1} \times\{1\}\right)$ is not an accidental peripheral, then it is freely homotopic into $\partial M$ in $S$. So, by cutting $N\left(A ; S^{1} \times[0,1]\right)$ and pasting a parallel copy of the free homotopy in $S$, we obtain a map $f^{\prime}: S^{1} \times[0,1] \rightarrow M$ with $\left|f^{\prime-1}(S)\right|<\left|f^{-1}(S)\right|$, a contradiction to the minimality of $\left|f^{-1}(S)\right|$.

Let $M^{\prime}$ be the cutting result $M-\operatorname{int} N(S)$, and let $S^{+}$be the component of $\partial N(S)$ with $S^{+} \cap f(A) \neq \emptyset$. Since each component of $\partial M$ is a torus and $S$ is essential, $M^{\prime}$ forms a sutured manifold. Set $T^{\prime}=\hat{N}\left(\operatorname{Im}(f) \cap \partial M^{\prime} ; \partial M^{\prime}\right)$ where $\hat{N}\left(\operatorname{Im}(f) \cap \partial M^{\prime} ; \partial M^{\prime}\right)$ is the union of $N\left(\operatorname{Im}(f) \cap \partial M^{\prime} ; \partial M^{\prime}\right)$ and the disks bounded by $\partial N\left(\operatorname{Im}(f) \cap \partial M^{\prime} ; \partial M^{\prime}\right)$ in $\partial M^{\prime}$. Then $T^{\prime}$ is incompressible in $M^{\prime}$, so the pair $\left(M^{\prime}, T^{\prime}\right)$ forms a Haken-manifold pair.

Since $f\left(S^{1} \times\{1\}\right)$ is in a suture, the component of $\hat{N}\left(\operatorname{Im}(f) \cap \partial M^{\prime} ; \partial M^{\prime}\right)$ which contains $f\left(S^{1} \times\{1\}\right)$ is an annulus. By applying the Annulus theorem ([6, VIII.10]) to $\left(M^{\prime}, T^{\prime}\right)$ we get a well-embedded Seifert pair $(\Sigma, \Phi) \subset\left(M^{\prime}, T^{\prime}\right)$. If the component $\left(\Sigma^{\prime}, \Phi^{\prime}\right) \subset(\Sigma, \Phi)$ that contains $\operatorname{Im}(f)$ is an $I$-pair, then it has to be $\left(\left(S^{1}\right) \times I\right) \times I$ and either get an embedded accidental annulus or actual peripheral in $S$. Hence we assume that $\left(\Sigma^{\prime}, \Phi^{\prime}\right)$ is an $S^{1}$-pair. If each component of $\partial \Phi^{\prime}$ is parallel to $\partial S^{+}$ in $S^{+}$, the loop $l$ is also parallel to $\partial S^{+}$in $S^{+}$. So, some component of $\operatorname{Fr} \Sigma^{\prime}$ is an embedded accidental annulus for $S^{+}$. Since $S$ is parallel to $S^{+}$in $M$, the embedded accidental annulus can be modified to an embedded accidental annulus for $S$.

A surface $F$ properly embedded in a 3 -manifold $M$ is $\pi_{1}$-essential if $\partial N(F)$ is incompressible and $\partial$-incompressible in $M-\operatorname{int} N(F)$. We will deal with one-sided surfaces, so we prove the following lemma needed later. 
Lemma 2.2. Let $K$ be a non-trivial knot in $S^{3}$, and let $S$ be a properly embedded, connected, one-sided surface in $E(K)$. Then, $S$ is $\pi_{1}$-essential if and only if $\partial N(S ; E(K))$ is incompressible in $E(K)$.

Proof. Suppose $\partial N(S)$ is incompressible in $E(K)$. First we claim that if $\partial N(S)$ is $\partial$ compressible in $E(K)$, then $\partial N(S)$ is $\partial$-parallel into $\partial E(K)$ by the irreducibility of $E(K)$ and the incompressibility of $\partial N(S)$. To see this, let $D$ denote a $\partial$-compressing disk of $\partial N(S)$ in $E(K)$ and let $A$ be the annular component of $\partial E(K)-N(S)$ which meets $\partial D$. Let $D_{+}, D_{-}$be components of $\partial N(D ; E(K)-\operatorname{cl}(N(S)))$ which are parallel copies of $D$, and let $D_{*}$ be the "rectangular" component of $A-\left(D_{+} \cup D_{-}\right)$ which does not meet $\partial D$. Put $D^{\prime}=D_{*} \cup D_{+} \cup D_{-}$. Observe that the surface $D^{\prime}$ is a disk with $\partial D^{\prime} \subset \partial N(S)$ and by the incompressibility, $\partial D^{\prime}$ bounds a disk $D^{\prime \prime}$ in $\partial N(S)$. Hence the component of $\partial N(S)$ having the $\partial$-compressing disk $D$ is an annulus consisting of $D^{\prime \prime}$ and $N(\partial D \cap \partial N(S) ; \partial N(S))$. Since the knot exterior $E(K)$ is irreducible, the sphere $D^{\prime \prime} \cup D^{\prime}$ bounds a 3-ball $B$ in $E(K)$. Thus, $B \cup N(D)$ forms a solid torus with meridian disk $D$, so this gives a $\partial$-parallelism.

Notice that the $\partial$-compressing disk is in $\operatorname{cl}(E(K)-N(S))$ since for a twisted $I$ bundle over surface, the corresponding $\partial I$-bundle is $\partial$-incompressible in the twisted $I$-bundle. So, $\partial N(S)$ is an annulus parallel into $\partial E(K)$. Thus the one-sided surface $S$ is homeomorphic to a Möbius band and $\pi_{1}(E(K))=\pi_{1}(S)=\mathbb{Z}$. This means that $K$ is trivial.

Proof of Theorem 1.3. Let $S_{0}$ be a connected $\pi_{1}$-essential surface with boundary slope $\gamma$, and let $A_{0}$ be an accidental annulus for $S_{0}$. By Lemma 2.1 we may assume the accidental annulus $A_{0}$ is embedded. We construct (possibly non-orientable) $\pi_{1}$ essential surfaces $\left\{S_{i}\right\}$ inductively as follows. We are given a $\pi_{1}$-essential surface $S_{i}$ and an accidental annulus $A_{i}$ for $S_{i}$. Let $B_{i}$ be the closure of the component of $\partial E(K)-\partial S_{i}$ which contains $\partial A_{i}$. We isotope the surface $S_{i} \cup B_{i}$ slightly into $\operatorname{int} E(K)$ and we set the resulting surface $S_{i+1}$. We put $A_{i+1}$ the closure of the component of $A_{i}-S_{i+1}$ which meets $\partial E(K)$ and we set $A_{i}^{\prime}$ the closure of the other component. We put $E\left(S_{i+1}\right)=\operatorname{cl}\left(E(K)-N\left(S_{i+1}\right)\right)$. Here we denote $A_{i+1} \cap E\left(S_{i+1}\right)$ and $A_{i}^{\prime} \cap E\left(S_{i+1}\right)$ by the same symbols $A_{i+1}$ and $A_{i}^{\prime}$ respectively.

Proposition 2.3. The surface $S_{i+1}$ is $\pi_{1}$-essential in $E(K)$ and $A_{i+1}$ is an accidental annulus for $S_{i+1}$.

Proof of Proposition 2.3. Suppose there exists a compressing disk $D$ for $\partial N\left(S_{i+1}\right)$ in $E\left(S_{i+1}\right)$. Set $A=A_{i}^{\prime} \cup A_{i+1}$. We may assume that $D$ intersects $A$ transversely, and assume that the number $|D \cap A|$ is minimal among all compressing disks for $\partial N\left(S_{i+1}\right)$ in $E\left(S_{i+1}\right)$. If $|D \cap A|=0$, then we have $\partial D \subset \partial N\left(S_{i}\right)$, but this contradicts the $\pi_{1}$-essentiality of $S_{i}$.

We note that $A_{i}^{\prime}$ and $A_{i+1}$ are incompressible in $E\left(S_{i+1}\right)$. Otherwise, $K$ is trivial and $S_{0}$ must be a disk. This contradicts the accidentality of $S_{0}$. Hence, there is no loop in $D \cap A$ by the minimality of $|D \cap A|$.

Next, we will show that there exists no arc of $D \cap A$ which is inessential in $A$. For a contradiction, suppose that there is an $\operatorname{arc}$ of $D \cap A$ which is inessential in $A$. Let $\alpha$ be an arc of $D \cap A$ which is outermost in $A$, and $\delta$ the corresponding outermost disk in $A$. Cutting $D$ along $\alpha$ and pasting two copies of $\delta$ to them, we get two disks $D_{1}$ and $D_{2}$ properly embedded in $E\left(S_{i+1}\right)$. It follows from the essentiality of $\partial D$ in $\partial E\left(S_{i+1}\right)$ that at least one of $D_{1}$ and $D_{2}$ is a compressing disk for $\partial E\left(S_{i+1}\right)$ in 
$E\left(S_{i+1}\right)$ again. We exchange $D$ for the new compressing disk. However, $|D \cap A|$ strictly decreases; this contradicts the minimality of $|D \cap A|$.

Therefore, all arcs of $D \cap A$ are essential in $A$. Let $\alpha$ be an $\operatorname{arc}$ of $D \cap A$ which is outermost in $D$, and $\delta$ the corresponding outermost disk in $D$. Since one component of $\partial A_{i+1}$ is contained in $\partial E(K),\left|\delta \cap A_{i+1}\right|=0$. Now $\delta$ gives a $\partial$-compressing disk for $A_{i}^{\prime}$ in $E\left(S_{i+1}\right)$. When we recover $S_{i}$ from $S_{i+1}$ by an annulus compression along $A_{i+1}, \delta$ can be converted to a $\partial$-compressing disk for $A_{i}$ in $E\left(S_{i}\right)$, since $\delta \cap\left(E\left(S_{i+1}\right) \cap A_{i}^{\prime}\right)$ consists of one point. This contradicts the assumption that $A_{i}$ is an accidental annulus for $S_{i}$, and proves that $\partial N\left(S_{i+1}\right)$ is incompressible in $E(K)$.

If $S_{i+1}$ is one-sided, then it is $\pi_{1}$-essential by Lemma 2.2. If $S_{i+1}$ is two-sided and $\partial N\left(S_{i+1}\right)$ is $\partial$-compressible in $E(K)$, then $S_{i+1}$ is $\partial$-parallel annulus. By the construction of $S_{i+1}$, we have $\chi\left(S_{i+1}\right)=\chi\left(S_{i}\right)$ where $\chi$ denotes the Euler characteristic. It follows that $S_{i}$ is also an annulus, but $S_{i}$ cannot be accidental since all non-trivial loops are $\partial$-parallel. This proves that $\partial N\left(S_{i+1}\right)$ is $\partial$-incompressible in $E(K)$.

Now let us show that $A_{i+1}$ is an accidental annulus. It is noticed that $S_{i}$ is connected. If $A_{i+1} \cap S_{i+1}$ is parallel to $\partial S_{i+1}$ in $S_{i+1}$, then $S_{i}$ cannot be connected by the existence of the parallelism annulus in $S_{i+1}$. Hence, $A_{i+1}$ is an accidental annulus for $S_{i+1}$. This completes the proof of Proposition 2.3.

Since $\left|\partial S_{i+1}\right|=\left|\partial S_{i}\right|-2$, we have $\left|\partial S_{n}\right|=0$ or 1 for some integer $n$. If $\left|\partial S_{n}\right|=0$, we are done. If $\left|\partial S_{n}\right|=1$, the surface $S_{n+1}=\partial N\left(S_{n} \cup N(K)\right)$ is $\pi_{1}$-essential and accidental by the same argument as Proposition 2.3 since $\partial N\left(S_{n} ; E(K)\right)$ is $\pi_{1}$ essential and accidental. This proves Theorem 1.3

If $S_{0}$ is an accidental incompressible Seifert surface for $K$, by the argument in the proof of Theorem 1.3. $S_{1}=\partial\left(S^{3}-\operatorname{int} N\left(S_{0} ; S^{3}\right)\right)$ is incompressible. Hence $S_{0}$ is totally knotted. Thus, we have:

Proposition 2.4. An accidental incompressible Seifert surface $S$ is totally knotted and the knot complement contains a closed accidental incompressible surface of genus $2 g(S)$.

\section{Non-minimal genus totally knotted Seifert surfaces WITH NO ACCIDENTAL PERIPHERALS}

In this section, we show that there exists a knot with a totally knotted Seifert surface which is non-minimal genus and has no accidental peripherals.

Theorem 3.1 (non-minimal genus totally knotted Seifert surfaces). For any positive integer $n$, there exists a genus one knot such that it bounds a totally knotted Seifert surface of genus $n$.

The outline of the proof of Theorem 3.1 is the following. First, we will construct a knot $K$ on the boundary of a handlebody $V$ in $S^{3}$ such that $K$ is separating in $\partial V, \partial V-K$ is incompressible in $V$, and $K$ bounds a genus one Seifert surface in $V$. Next, re-embedding $(V, K)$ into $S^{3}$ so that $\partial V$ becomes to be incompressible in $S^{3}-\operatorname{int} V$, we obtain a totally knotted Seifert surface of genus $n>1$ as the closure of a component of $\partial V-K$. This follows a version of a result in [5].

Lemma 3.2 (cf. [5. Remark 2.1]). Let $K$ be a knot in $S^{3}$ which is contained in a closed surface $S$, and let $M_{1}$ and $M_{2}$ be closures of components of $S^{3}-S$. Suppose 
that $K$ is separating in $S, S-K$ is incompressible in $S^{3}-K$, and one of $M_{1}$ and $M_{2}$ is $\partial$-irreducible. Then both components of $S-K$ are totally knotted.

Proof. There exists an accidental surface $S^{\prime}$ in $\operatorname{int} M_{2}$ parallel to $S$. Hence by [5] Theorem 1.4 (A)], both components of $S-K$ are strongly essential, thus totally knotted.

Proof of Theorem 3.1. To construct a pair $(V, K)$ as above, let $K_{1}$ be a torus knot on the solid torus $V_{1}$ standardly embedded in $S^{3}$ and put $S_{1}=N\left(K_{1} ; \partial V_{1}\right)$. Let $K_{2}$ be a pretzel knot of type $(2,1,1, \cdots, 1)$, where the number of 1 is equal to $n$. Naturally, $K_{2}$ bounds a non-orientable surface with the first Betti number $n$ and its regular neighborhood is a genus $n$ handlebody, say $V_{2}$. We regard $K_{2}$ as a non-separating loop on $\partial V_{2}$. Then put $S_{2}=N\left(K_{2} ; \partial V_{2}\right)$. For each $S_{i}$, let $\alpha_{i}$ be an essential arc in $S_{i}$ and put $b_{i}=N\left(\alpha_{i} ; S_{i}\right)$. It is easily proved that $\partial V_{i}-K_{i}$ is incompressible in $V_{i}$ for $i=1,2$. Now, we perform a boundary-connected sum of $V_{1}$ and $V_{2}$ along $b_{1}$ and $b_{2}$ so that $S_{1}$ and $S_{2}$ are plumbed. Thus, we obtain a genus $n+1$ handlebody $V$, a knot $K$ on $\partial V$ as the boundary of the plumbed genus one Seifert surface $S_{1} \cup S_{2}$, and a genus $n$ Seifert surface $S$ for $K$ as the union of $S_{1}$ and $\partial V_{2}-S_{2}$.

To show that $\partial V-K$ is incompressible in $V$, we push $\operatorname{int}\left(S_{1} \cup S_{2}\right)$ into $\operatorname{int} V$ slightly. Let $\delta_{i}(i=1,2)$ be a $\partial$-compressing disk for $S_{i}$ in $V_{i}$ disjoint from $b_{i}$. If $S_{1} \cup S_{2}$ is compressible in $V$, then there exists a compressing disk $D$ for $S_{1} \cup S_{2}$ disjoint from $\delta_{1} \cup \delta_{2}$. Hence $D$ is contained in $V_{1}$ or $V_{2}$, and hence $S_{i}$ is compressible in $V_{i}(i=1$ or 2$)$. This shows that $K_{i}$ is trivial, a contradiction.

Now, suppose that $\partial V-K$ is compressible in $V$ and let $D^{\prime}$ be a compressing disk. Since $S_{1} \cup S_{2}$ is incompressible in $V$ by the above argument, $D^{\prime}$ can be chosen so that $D^{\prime} \cap\left(S_{1} \cup S_{2}\right)=\emptyset$. Moreover, cutting and pasting along an outermost disk of $\delta_{i}, D^{\prime}$ can be chosen so that $D^{\prime} \cap\left(\delta_{1} \cup \delta_{2}\right)=\emptyset$. However, this contradicts that $\partial V_{i}-K_{i}$ is incompressible in $V_{i}$.

Next, we re-embed $(V, K)$ so that $\partial V$ becomes incompressible in $S^{3}-\operatorname{int} V$. Then Lemma 3.2 assures us that $S$ is totally knotted. This completes the proof of Theorem 3.1 .

Proposition 3.3. Let $K$ be a knot in $S^{3}$ which is contained in a closed surface $S$, and let $M_{1}$ and $M_{2}$ be the closures of components of $S^{3}-S$. Suppose that $K$ is separating in $S, S-K$ is incompressible in $S^{3}-K$, and one of $M_{1}$ and $M_{2}$ is $\partial$ irreducible and anannular (contains no properly embedded essential annuli). Then both components of $S-K$ are totally knotted Seifert surfaces without accidental peripherals.

Proof of Proposition 3.3. Let $S_{1}$ and $S_{2}$ be components of $S-K$. Then, by Lemma 3.2, both $S_{1}$ and $S_{2}$ are totally knotted.

Suppose without loss of generality that $S_{1}$ is accidental, and let $A$ be an accidental annulus for $S_{1}$. We may assume that $N(\partial A ; A) \subset M_{1}$ or $M_{2}$, int $A$ intersects $S_{2}$ transversely, and assume that $\left|\operatorname{int} A \cap S_{2}\right|$ is minimal up to isotopy of $A$. By the supposition, without loss of generality, we may assume that $M_{2}$ is $\partial$-irreducible and anannular. If $\operatorname{int} A \cap M_{2} \neq \emptyset$, then the minimality of $\left|\operatorname{int} A \cap S_{2}\right|$ guarantees that $M_{2}$ contains an essential annulus, but this is a contradiction. Hence, $\operatorname{int} A$ is contained in $M_{1}$. Since $\pi_{1}\left(S^{3}\right)$ is trivial, $S$ must be compressible in $M_{1}$. Let $D$ be a compressing disk for $S$ in $M_{1}$, and assume that $|D \cap A|$ is minimal over all compressing disks. By the argument similar to the proof of Theorem 1.3, all arcs 


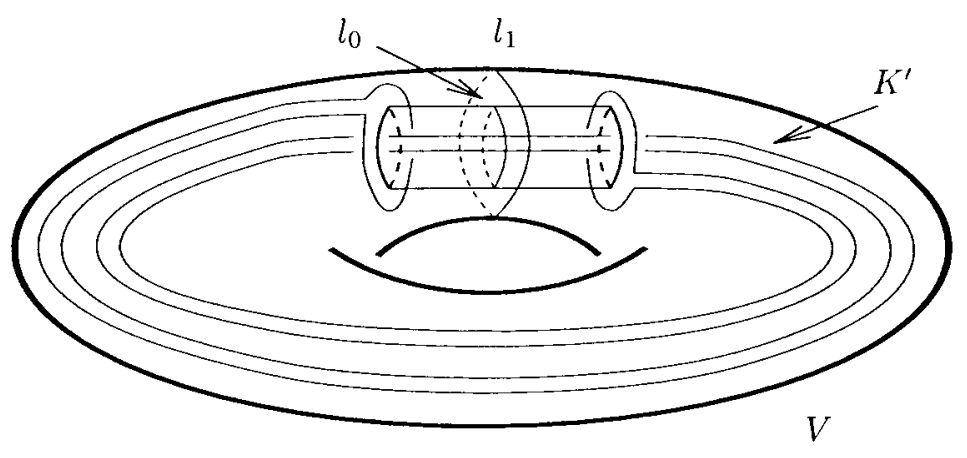

FIGURE 1.

of $D \cap A$ are essential in $A$, and there exists an outermost disk $\delta$ of $D$ which gives a $\partial$-compressing disk for $A$ in $M_{1}$. Then $\delta \cap \partial M_{2}$ is an arc in $S_{1}$ connecting $K$ and the accidental peripheral, since $K$ is separating in $S$. This shows that $A$ is parallel to an annulus in $S$ and contradicts the definition of $A$.

In section2, we showed that an incompressible accidental Seifert surface is totally knotted (Proposition 2.4). But the contrary does not hold. In fact, in the proof of Theorem 3.1, if one re-embeds $(V, K)$ into $S^{3}$ so that $\partial V$ is incompressible and anannular in $S^{3}-\operatorname{int} V$, then the resulting totally knotted Seifert surfaces are not accidental by Proposition 3.3

\section{Accidental totally knotted Seifert surface}

In this section, we prove Theorem 1.5 by constructing infinitely many knots in $S^{3}$ of distinct types with accidental incompressible Seifert surfaces. Also we construct a closed hyperbolic Haken 3-manifold which contains a hyperbolic knot with an accidental incompressible Seifert surface.

Proof of Theorem 1.5. Let $V$ be a solid torus and $K^{\prime}$ the knot in $V$ as shown in Figure 1, and let $S_{0}$ be the genus one Seifert surface spanned by $K^{\prime}$ in $V$.

Let $K_{0}$ be a composite knot in $S^{3}$, and for any integer $n>0$, let $A_{0}, A_{1}, \cdots, A_{n}$ be mutually parallel essential annuli in $E\left(K_{0}\right)$ coming from the decomposing sphere of the composite knot $K_{0}$. There exists an annulus $A$ in $V$ such that $\partial A=l_{0} \cup l_{1}$ and $A \cap S_{0}=l_{0}$ where $l_{0}$ is a non-separating curve in $S_{0}$ and $l_{1}$ is the boundary of a meridian disk of $V$. Let $N$ be a regular neighbourhood of $\partial\left(V-\operatorname{int} N\left(S_{0}\right)\right)-\partial V$ in $V-\operatorname{int} N\left(S_{0}\right)$ which is homeomorphic to a product $S^{*} \times I$ where $S^{*}$ is a closed surface with $\chi\left(S^{*}\right)=2 \chi\left(S_{0}\right)$ and $S^{*} \times\{1\} \subset \operatorname{int} V$. Set $S_{i}=S^{*} \times\{i / n\}$ for $1 \leq i \leq n$. After " $\partial$-annulus-compressions" along $A$ (see Figure 2), we get surfaces $S_{n}^{\prime}, S_{n-1}^{\prime}, \cdots, S_{0}^{\prime}$ from the surfaces $S_{n}, S_{n-1}, \cdots, S_{0}$. We remark that $K^{\prime}$ is a non-trivial knot, and each $S_{i}^{\prime}$ is not homeomorphic to an annulus.

Proposition 4.1. Each $S_{i}^{\prime}$ is $\pi_{1}$-essential in $V-K^{\prime}$.

Proof. First, we claim that it suffices to show $S_{i}$ is incompressible in $V-K^{\prime}$. If $D$ is a compressing disk for $S_{i}^{\prime}$, then $D$ can be modified to a compressing disk for $S_{i}$. Hence, if $S_{i}$ is incompressible, then $S_{i}^{\prime}$ is also incompressible. Suppose $D$ is a $\partial$ compressing disk for $S_{i}^{\prime}$ such that $\partial D=\alpha \cup \beta, \alpha \subset \partial V, \beta \subset S_{i}^{\prime}$. By the construction, 


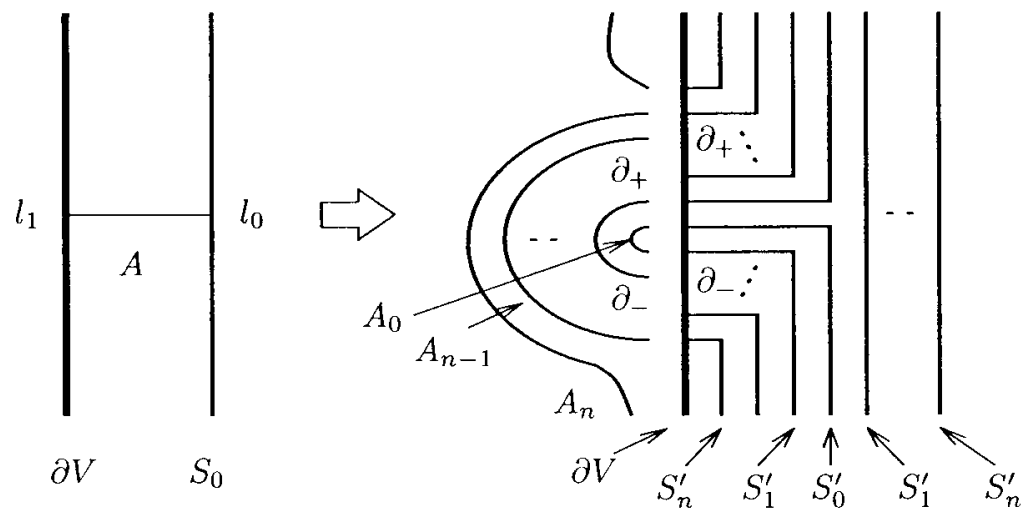

FiguRE 2.

$\partial S_{i}^{\prime}$ separates $\partial V$ into a union of two annuli $B_{0}, B_{1}$, so $\alpha$ is an essential arc in, say, $B_{0}$. We identify a regular neighbourhood $N^{\prime}$ of $D$ in the closure of $V-S_{i}^{\prime}$ with $D \times I$. Put $D_{i}=D \times i$ for $i=0,1$. Then $\left(B_{0}-N^{\prime}\right) \cup D_{0} \cup D_{1}$ forms a disk $D^{\prime}$ such that $\partial D^{\prime} \subset S_{i}^{\prime}$. If $S_{i}^{\prime}$ is incompressible, then $\partial D^{\prime}$ bounds a disk $E$ in $S_{i}^{\prime}$ on the side not containing $\partial D$ and the sphere $D^{\prime} \cup E$ bounds a 3-ball $C$ on the side not containing $D$. The solid torus $C \cup N^{\prime}$ is a $\partial$-parallelism for $S^{\prime}$. This is a contradiction and proves our first claim.

Next, we show that $S_{i}$ is incompressible. Since $S_{0}$ is a genus one Seifert surface and $K^{\prime}$ is non-trivial, it is incompressible. Put $F_{0}=\partial N\left(S_{0} ; V\right)$. Since $F_{0}$ is incompressible in $N\left(S_{0} ; V\right)-K^{\prime}$, it suffices to show that $F_{0}$ is incompressible in $V-\operatorname{int} N\left(S_{0} ; V\right)$. Let $D^{\prime}$ be a meridian disk of $V$ which contains $A$ such that $\partial D^{\prime}=l_{1}$ and $D^{\prime}-\operatorname{int} N\left(S_{0} ; V\right)$ is a union of annuli $B, B^{\prime}\left(B^{\prime} \subset A\right)$. Let $D$ be a compressing disk for $F_{0}$. We assume $\left|D \cap\left(B \cup B^{\prime}\right)\right|$ is minimal among all compressing disks. We claim that if $D$ intersects $B \cup B^{\prime}$, then $D \cap B$ is a union of essential $\operatorname{arcs}$ in $B$, and $D \cap B^{\prime}=\emptyset$. If some component is an inessential loop or arc, we can reduce the number $\left|D \cap\left(B \cup B^{\prime}\right)\right|$. Let $\Delta$ be an innermost disk of $D$ with respect to $D \cap\left(B \cup B^{\prime}\right)$. Then $\partial \Delta$ is a core of $B$ or $B^{\prime}$. But there exists a loop $l$ in $N\left(S_{0} ; V\right)$ such that $l$ intersects the sphere $D^{\prime \prime} \cup \Delta$ with non-zero algebraic intersection number, where $D^{\prime \prime}$ is a disk in $D^{\prime}$ bounded by $\partial \Delta$. This means that $D^{\prime \prime} \cup \Delta$ does not bound a 3-ball, a contradiction to the irreducibility of $V$. If $\Delta^{\prime}$ is an outermost disk in $D$ with $\partial \Delta^{\prime}=\alpha^{\prime} \cup \beta^{\prime}\left(\alpha^{\prime} \subset B\right)$, then by the above claim, $\alpha^{\prime}$ is an essential arc in $B$. If $F=F_{0}-\operatorname{int} N\left(D^{\prime} ; V\right)$, then $F \cap \beta^{\prime}$ is a connected arc in $F$ since int $\beta^{\prime} \cap\left(B \cup B^{\prime}\right)=\emptyset$. But it is impossible because two points $\partial\left(\beta^{\prime} \cap F\right)$ lie in distinct components of $F$. So, $D \cap\left(B \cup B^{\prime}\right)=\emptyset$. Now, it is easy to see that $\partial D$ bounds a disk in $F$. This completes the proof.

We embed $V$ in $S^{3}$ so that $V=N\left(K_{0}\right), \partial_{+} A_{0}=\partial_{-} S_{0}^{\prime}, \partial_{+} A_{i}=\partial_{+} S_{i-1}^{\prime}(1 \leq i \leq$ $n$ ), $\partial_{-} A_{n}=\partial_{+} S_{n}^{\prime}$, and $\partial_{-} A_{i}=\partial_{-} S_{i+1}^{\prime}(n-1 \geq i \geq 0)$ (see Figure 21). We let $K$ be the image of $K^{\prime}$.

The surface $S=\bigcup_{i=0}^{n} A_{i} \cup S_{i}^{\prime}$ is an orientable Seifert surface for $K$ of genus $n+1$, and has an accidental peripheral (Figure 3 ). 


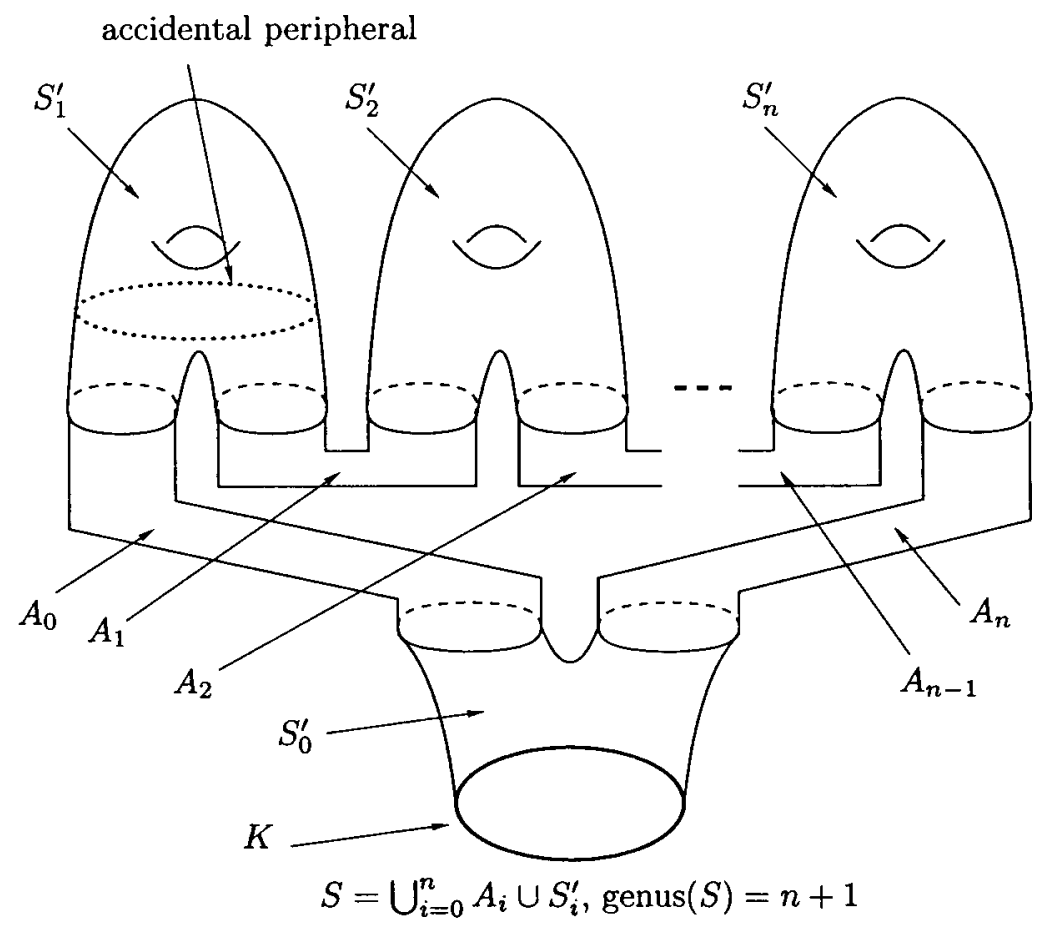

FIGURE 3.

Since $K_{0}$ is composite, each annulus $A_{i}$ is $\pi_{1}$-essential in $E\left(K_{0}\right)$, and by Proposition 4.1 each $S_{i}^{\prime}$ is $\pi_{1}$-essential. Hence we can show that the Seifert surface $S$ is incompressible in $E(K)$.

It is not hard to see, because $S_{i}^{\prime}$ is incompressible, that the wrapping number $w_{V}\left(K^{\prime}\right)=2$ where the wrapping number $w_{V}\left(K^{\prime}\right)$ is defined to be the geometric intersection number of $K^{\prime}$ with a meridian disk in $V$. So, by twisting the knot $K^{\prime}$ along the loop $l_{1}$ in the solid torus $V$, one can produce infinitely many knots of distinct types, by [7, Theorem 2.1]. This completes the proof.

Remark 4.2. In 8 and [4, genus one knots with arbitrarily high genus Seifert surface are given. In particular, the knot Gustafson ([4]) constructed is simple.

The accidental Seifert surface constructed above is actually non-minimal genus, as the result of Fenley [2. Here we remark that a minimal genus Seifert surface for a knot in some 3-manifold can be accidental.

Proposition 4.3. There exists a closed hyperbolic Haken 3-manifold $M$ such that $M$ contains a hyperbolic knot $K$ with an accidental Seifert surface of genus one.

Proof. Let $T$ be a genus one, orientable surface with a connected boundary. Let $l$ be an essential simple closed curve in $T$. Put $M_{0}=T \times I, H_{+}=\partial T \times I$, and $H_{-}=$ $N(l ; T) \times\{1\}$. By identifying two annuli $H_{+}$and $H_{-}$with some homeomorphism, we obtain an orientable 3-manifold $M_{1}$ with $\partial M_{1}$ connected, closed, genus two. Using the product structure of $M_{0}$, it can be shown that each component of $\partial M_{0}-$ $\left(\partial H_{+} \cup \partial H_{-}\right)$is incompressible, there is no properly embedded disk $D$ in $M_{0}$ such 
that each of $\partial D \cap H_{ \pm}$and $\partial D \cap\left(\partial M_{0}-\operatorname{int} H_{+} \cup \operatorname{int} H_{-}\right)$is a single arc, and that there is no essential annulus with boundaries in $H_{+} \cup H_{-}$. Thus, $M_{1}$ is irreducible, $\partial$-irreducible, and atoroidal.

Put $K=\partial T \times\{1 / 2\}$. Notice that the knot $K$ bounds a genus one Seifert surface, still denoted by $T$, with an accidental annulus $l \times[1 / 2,1]$ in $M_{1}$. Let $V$ be an irreducible, $\partial$-irreducible, atoroidal, and anannular 3-manifold such that $\partial V$ is a genus two closed surface.

We glue $M_{1}$ and $V$ with their boundaries, and get a closed manifold $M$ which is irreducible, atoroidal, and contains an incompressible surface, say the gluing surface. Thus, this manifold is hyperbolic by Thurston's geometrization theorem (cf. [9]).

To see that the knot $K$ is hyperbolic, we show that $E(K)$ is irreducible, $\partial$ irreducible, atoroidal, and anannular. Using the product structure again, we can show that $E\left(K ; M_{1}\right)=M_{1}-\operatorname{int} N\left(K ; M_{1}\right)$ is irreducible, $\partial$-irreducible, and atoroidal. Since $V$ is irreducible, $\partial$-irreducible, atoroidal, and anannular, the exterior $E(K ; M)=V \cup_{\partial V=\partial M_{1}} E\left(K ; M_{1}\right)$ is irreducible, $\partial$-irreducible, atoroidal and anannular.

\section{ACKNOWLEDGMENT}

We thank Dr. Kazuhiro Ichihara for suggesting the problem and for his kind advice and useful comments.

\section{REFERENCES}

1. M. Culler, C. Gordon, J. Luecke, and P. Shalen, Dehn surgery on knots, Ann. of Math. 125 (1987), 237-300. MR 89c:57015

2. S. R. Fenley, Quasi-Fuchsian Seifert surfaces, Math. Z. 228 (1998), 221-227. MR 99c:57037

3. D. Gabai, Foliations and the topology of 3-manifolds III, J. Diff. Geom. 26 (1987), 479-536. MR 89a:57014b

4. R. F. Gustafson, A simple genus one knot with incompressible spanning surfaces of arbitrarily high genus, Pacific J. Math. 96 (1981), 81-98. MR 83a:57008

5. K. Ichihara and M. Ozawa, Accidental surfaces in knot complements, J. Knot Theory and its Ramifications, 9 (2000), 725-733. MR 2001f:57007

6. W. Jaco, Lectures on Three Manifold Topology, AMS Conference board of Math. No. 43, 1980. MR 81k:57009

7. M. Kouno, K. Motegi and T. Shibuya, Twisting and knot types, J. Math. Soc. Japan 44 (1992), 199-216. MR 93e:57011

8. H. C. Lyon, Incompressible surfaces in knot spaces, Trans. Amer. math. soc, 157 (1971), 53-62. MR 43:1169

9. W. P. Thurston, The geometry and topology of 3-manifolds, Lecture notes, Princeton University, 1978.

Department of Mathematics, School of Education, Waseda University, Nishiwaseda 1-6-1, ShinJuku-Ku, TOKYO 169-8050, JAPAN

E-mail address: ozawa@musubime.com

Current address: Natural Science Faculty, Faculty of Letters, Komazawa University, 1-23-1

Komazawa, Setagaya-ku, Tokyo, 154-8525, Japan

Department of Mathematics, Keio University, Hiyoshi 3-14-1, Kohoku-Ku, Yokohama 223-8522, JAPAN

E-mail address: yukihiro@math.keio.ac.jp 